Alexander Dix, Berliner Beauftragter für Datenschutz und Informationsfreiheit

\title{
Safe Harbor vor dem Aus?
}

Das von Anfang an umstrittene Safe Harbor-Abkommen von 2000 steht möglicherweise vor dem endgültigen Aus. Es war ein Kompromiss, der von den Datenschutzbeauftragten und vom Europäischen Parlament stets kritisiert wurde. Das zwischen der US-Regierung und der Europäischen Kommission geschlossene Übereinkommen entspricht in mehreren Punkten nicht dem europäischen Datenschutzstandard, etwa bezüglich der Zweckbindung, des eingeschränkten Auskunftsrechts Betroffener und der Weiterübermittlung in Drittstaaten. Das zentrale Defizit des Abkommens liegt bei der Verifikation. Nach wie vor genügt eine einfache Mitteilung an das US-Handelsministerium, mit der sich jedes Unternehmen (mit Ausnahme bestimmter Branchen) selbst ein angemessenes Datenschutzniveau nach den Safe Harbor-Grundsätzen attestieren kann. Ob das im Zeitpunkt der Meldung zutrifft, wird nicht überprüft. Die Federal Trade Commission (FTC) hat zwar den Auftrag, die spätere Einhaltung der Safe Harbor-Grundsätze zu überprüfen und sie tut dies auch inzwischen verstärkt, nachdem zuvor in mehreren Studien eklatante Vollzugsdefizite festgestellt worden waren. Die Prüfkompetenz der FTC beschränkt sich aber auf die Bekämpfung der Irreführung von Verbrauchern, die darin liegen kann, dass ein Safe-Harbor-Unternehmen seine Selbstzertifizierung nicht - wie vorgeschrieben - jährlich erneuert oder bei der Datennutzung nicht das einhält, was es den Konsumenten versprochen hat. In solchen Fällen hat die FTC allerdings in den letzten Jahren teilweise hohe Bußgelder verhängt. Allerdings gehört der Beschäftigtendatenschutz, der in den USA ohnehin praktisch nicht existiert, nicht zum Zuständigkeitsbereich der FTC. In der Praxis berufen sich aber viele US-Konzernmütter mit Töchtern in Deutschland und Europa bei der Übermittlung von Beschäftigtendaten auf die Grundsätze des Safe Harbor.

Die Europäische Kommission hat - gerade um den Bedenken der Datenschutzbeauftragten und des Europäischen Parlaments Rechnung zu tragen - in ihre Entscheidung zum Safe Harbor einen doppelten Vorbehalt zugunsten der nationalen Datenschutzbehörden aufgenommen. Diese haben zunächst zu prüfen, ob die Datenverarbeitung auf der „ersten Stufe“ nach nationalem Datenschutzrecht zulässig ist. Damit soll verhindert werden, dass das nationale Datenschutzrecht durch den Export in die USA umgangen wird. Andererseits hat die Kommission mit dem Safe Harbor-Abkommen prinzipiell anerkannt, dass US-Unternehmen, die sich diesen Grundsätzen unterwerfen, ein angemessenes Datenschutzniveau nach den europäischen Vorschriften zum Datenexport gewährleisten. Sie sind also prinzipiell mögliche Importeure von Personendaten aus Europa. Wenn die deutschen und europäischen Aufsichtsbehörden allerdings zu dem Schluss kommen, dass eine „hohe Wahrscheinlichkeit“ besteht, dass die Safe
Harbor-Grundsätze nicht eingehalten werden und dadurch das „unmittelbar bevorstehende Risiko eines schweren Schadens für die betroffenen Personen" geschaffen würde, können sie konkrete Übermittlungen von Deutschland in die USA vorübergehend aussetzen. Die Aussetzung ist zu beenden, sobald sichergestellt ist, dass die Safe Harbor Grundsätze wieder eingehalten werden (Art. 3 der Kommissions-Entscheidung v. 26.7.2000 zu Safe Harbor). Entsprechende Vorbehalte finden sich im übrigen auch in den Entscheidungen der Kommission zu den Standardvertragsklauseln von 2001 und 2010.

Die Art. 29-Gruppe der europäischen Datenschutzbeauftragten hat in mehreren Stellungnahmen 2014 betont, dass die im Safe Harbor-Abkommen wie auch in den Entscheidungen zu den Standardvertragsklauseln enthaltenen Vorbehalte zugunsten der Sicherheitsbehörden in keinem Fall die anlasslose und flächendeckende Überwachungspraxis der NSA und anderer Geheimdienste rechtfertigt, die Edward Snowden öffentlich gemacht hat. Sie überschreitet das Maß dessen, was nach der Europäischen Menschenrechtskonvention in einer demokratischen Gesellschaft notwendig ist. Selbst der Google-Chef Larry Page hat in diesem Zusammenhang formuliert: „Ich glaube, es ist mit demokratischen Prinzipien unvereinbar, wenn wir die Nutzer vor Regierungshandeln schützen müssen, von dem nie die Rede war.“

Deutsche Aufsichtsbehörden prüfen gegenwärtig in Berlin und Bremen, ob sie den transatlantischen Datenverkehr in bestimmten Einzelfällen aussetzen sollen. Die Verfahren sind noch nicht abgeschlossen. Die Berliner Aufsichtsbehörde hat allerdings schon zwei Unternehmen davon überzeugen können, ihre Datenverarbeitung nicht an marktbeherrschende Anbieter in den USA auszulagern, ohne dass es dazu einer Anordnung bedurft hätte.

Wenn es nicht gelingt, zwischen Europa und den USA in Kürze (die Europäische Kommission will die Verhandlungen bis Mai 2015 abschließen) zu einer politischen Einigung über die nachprüfbare Datensammlung durch US-Nachrichtendienste zu kommen und darüber hinaus die übrigen Mängel des Safe Harbor-Abkommens zu beheben, muss die Kommissionsentscheidung zu Safe Harbor aufgehoben werden. Es ist bezeichnend, dass der Entwurf für eine Datenschutz-Grundverordnung nicht nur kein Äquivalent für Safe Harbor enthält, sondern nach dem Willen des Parlaments darüber hinaus den Zugriff ausländischer Behörden auf Datenbestände in Europa an zusätzliche Bedingungen knüpfen soll. Aber auch die europäischen Staaten müssen die Datenerhebung durch die eigenen Geheimdienste begrenzen und kontrollierbar gestalten. Generell gilt: Exzessive Überwachung durch unzureichend kontrollierte Geheimdienste gefährdet das Datenschutzniveau und den freien Datenverkehr weltweit. 